\title{
Investigation of Dust-Ion Acoustic Waves in a Magnetized Collisional Dusty Plasma with Kappa Distribution Function for Electrons
}

\author{
Hossein Zahed ${ }^{a, *}$, Mohammad Reza Sayyar,c, Sayyed Jalal Pestehe ${ }^{b}$, and Samad Sobhanian ${ }^{b}$ \\ aDepartment of Physics, Sahand University of Technology, Tabriz, Iran \\ bDepartment of Atomic and Molecular Physics, University of Tabriz, Tabriz, Iran \\ 'Faculty of Science, University of Maragheh, Maragheh, Iran
}

*Corresponding author' Email: Zahed@sut.ac.ir

Received: Aug. 16, 2016, Revised: Jan. 29, 2017, Accepted: Apr. 26, 2017, Available Online: Dec. 1, 2018

DOI: 10.29252/ijop.12.2.81

\begin{abstract}
The propagation of arbitrary amplitude dust ion acoustic waves (DIAWs) in a magnetized collisional dusty plasma including hot electrons, with kappa velocity distribution for electrons, warm ions and dust particles has been studied. In the presence of immobile massive dust particulates, DIAWs have been investigated through the Sagdeev pseudopotential method. It is demonstrated that the amplitude and width of the pseudo-potential are increased with the ion density and also with Directional cosines. It is shown that the behaviors of the amplitude and the width of the wave in terms of all of plasma parameters is similar to the our recently work, and the spectral index has a little effect on the wave.
\end{abstract}

KEYWORDS: magnetized dusty plasma, Dust ion acoustic waves, Sagdeev pseudo-potential method.

\section{I.INTRODUCTION}

Nowadays, the study of dusty plasmas properties has received a great deal of attention both theoretically and experimentally. In the presence of massive and highly charged dust particulates in an usual electron ion plasma new types of waves, such as dust acoustic (DA) waves and dust-ionacoustic (DIA) waves are excited. These dusts can be regarded as static or mobile particles [1]. The nonlinear waves particularly, the DIA solitary waves (DIASW) have been theoretically investigated by several authors [2-6].
In the most of the above mentioned investigations, Maxwellian or Maxwellian Boltzmann distribution functions have been used. However, a lot of theoretical observations of space plasmas are often characterized by a non- Maxwellian particle distribution function. In these plasmas, superthermal particles which are produced due to the effect of wave particle interaction or external forces. Superthermal plasmas are relativistic pulsar wind, solar wind, magnetosphere, interstellar medium, auroral zone plasmas, plasmas produced during an ultra-intense laser pulse interaction with matter. [7, 8]. These kinds of a plasma, can be characterized by generalized Lorentzian or kappa distribution function [9, 10]. This Lorentzian (kappa) velocity distribution is used to model the electrons in magnetosphere and electromagnetic ion cyclotron waves in equatorial ring protons $[11,12]$. Using a kappa distribution for plasma particles, many authors have studied the propagation of ion acoustic waves in a magnetized plasma using Sagdeev potential method. The one dimensional kappa velocity distribution is

$$
F_{\kappa}(v)=\frac{1}{\left(\pi \kappa \theta^{2}\right)^{3 / 2}} \frac{\Gamma(\kappa+1)}{\Gamma(\kappa-1 / 2)}\left(1+\frac{v^{2}}{\kappa \theta^{2}}\right)^{-(\kappa+1)}
$$

where $\theta$ is the most probable speed (effective thermal speed), related to the usual thermal 
velocity

$$
V_{t}=\left(K_{B} T / m\right)^{1 / 2}
$$
$\theta=[(2 \kappa-3) / \kappa] V_{t}, T$ is the characteristic kinetic temperature, $\kappa$ is spectral index and $K_{B}$ is the Boltzmann constant. The most probable speed, and hence the $\kappa$-distribution, is defined for $\kappa>3 / 2[7,8,11]$. Nonlinear propagation of ion acoustic waves in a magnetized plasma, electron acoustic waves in a two temperature electron plasma and linear and nonlinear propagation of these sound waves again with the presence of hot electron component and so on have been already studied [13].

Recently, we have investigated the propagation of dust ion acoustic wave in a dusty plasma in the presence of nonthermal electrons [14]. In this paper, the previous work has been extended with the warm ions and hot electrons. In the second section, the governing basic equations are presented similar to our previous paper [14]. The resultant dispersion relation and as well as the Sagdeev potential are derived in the third section. Finally, the numerical investigations are presented in the last section.

\section{BASIC EQUATIONS}

The continuity and momentum equations for ions and also Poisson equation are:

$$
\begin{aligned}
& \partial_{t} n_{i}+\nabla \cdot\left(n_{i} \mathbf{v}_{i}\right)= 0, \\
& \partial_{t} \mathbf{v}_{i}+\left(\mathbf{v}_{i} \cdot \nabla\right) \mathbf{v}_{i}= \frac{e}{m_{i}}\left(\mathbf{E}+\frac{B_{0} \mathbf{v}_{i} \times \mathbf{e}_{\mathbf{z}}}{c}\right) \\
&-\nabla \mathbf{p}_{i}-m_{i} n_{i} \mathbf{v}_{i} v, \\
& \nabla^{2} \varphi=-4 \pi\left[-e n_{e}+e n_{i}-e z_{d} n_{d}\right],
\end{aligned}
$$

where $n_{e}, n_{i}$, and $n_{d}$ are electron, ion, and dust densities respectively. $\mathbf{v}_{i}, v$, and $m_{i}$ indicating the ion velocity, collision frequency and its mass. $\mathrm{qd}=-$ ezd where $\varphi$ is the plasma potential, $z_{d}$ is the dust charge number, so that the charge of the dust is given by $q d=-e z d$, with e being the elementary charge. Here, electrons are assumed to be kappa distributed and the expression for the electron density is as follows: $n_{e}=\frac{1}{\delta_{1}}\left(1-\frac{\varphi}{\kappa-3 / 2}\right)^{-(\kappa-1 / 2)}$,

where, $\delta_{1}=\frac{n_{i 0}}{n_{e 0}}$.

We assume that the wave is propagating in the $\mathrm{X}-\mathrm{Z}$ plane. After normalization, the above system of equations is reduced to:

$$
\begin{aligned}
& \partial_{t} n+\partial_{x}\left(n v_{x}\right)+\partial_{z}\left(n v_{z}\right)=0 \\
& \partial_{t} v_{x}+\left(v_{x} \partial_{x}+v_{z} \partial_{z}\right) v_{x}=-\partial_{x} \varphi+v_{y} \\
& -\gamma \frac{\partial_{x} n}{n}+v v_{x}
\end{aligned}
$$

$\partial_{t} v_{y}+\left(v_{x} \partial_{x}+v_{z} \partial_{z}\right) v_{y}=-v_{x}-\gamma \frac{\partial_{y} n}{n}+v v_{y}$
$\partial_{t} v_{z}+\left(v_{x} \partial_{x}+v_{z} \partial_{z}\right) v_{z}=-\partial_{z} \varphi-\gamma \frac{\partial_{z} n}{n}+v v_{z}$

$\left(\partial_{x x}+\partial_{z z}\right) \varphi=$

$\beta\left[\frac{1}{\delta_{1}}\left(1-\frac{\varphi}{\kappa-3 / 2}\right)^{-(\kappa-1 / 2)}-\delta_{1} n+\delta_{2}\right]$

where, $\beta=r_{g}^{2} / \lambda_{e}^{2}, \delta_{1}=n_{i 0} / n_{e 0}, \delta_{2}=n_{d} z_{d} / n_{e 0}$. $r_{g}=C_{s} / \Omega \quad$ is the ion gyro radius and $\lambda_{e}=\left(T_{e} / 4 \pi n_{e 0} e^{2}\right)^{1 / 2}$ is the electron Debye length. $C_{S}=\left(T_{e} / m_{-}\right)^{1 / 2}$ is the ion acoustic velocity, and $\Omega=e B_{0} / m_{i} c$ is the ion gyro frequency. $n_{e 0}$ and $n_{i 0}$ are unperturbed electron and ion densities, respectively. The normalizations are:

$\Omega t \rightarrow t,\left(C_{s} / \Omega\right) \nabla \rightarrow \nabla, v_{i} / C_{s} \rightarrow v$, $n_{i} / n_{i 0} \rightarrow n$, and $e \varphi / T_{e} \rightarrow \varphi$,

To derive the dispersion relation for low frequency waves, we write the dependent variables as sum of equilibrium and perturbed parts. Assuming $n=1+\bar{n}, \quad v_{x}=\bar{v}_{x}, \quad v_{z}=\bar{v}_{z}$, $v_{y}=\bar{v}_{y}$, Eqs. (5) - (9) can be rewritten as:

$\partial_{t} \bar{n}+\partial_{x} \bar{v}_{x}+\partial_{z} \bar{v}_{z}=0$ 
$\partial_{t} \bar{v}_{x}=-\partial_{x} \bar{\phi}_{x}+\bar{v}_{y}-\gamma \frac{\partial_{x} \bar{n}}{1+\bar{n}}+v v_{x}$

$\partial_{t} \bar{v}_{y}=-v_{x}+v v_{y}$

$\partial_{t} \bar{v}_{z}=-\partial_{z} \bar{\phi}-\gamma \frac{\partial_{z} \bar{n}}{1+\bar{n}}+v v_{z}$

$$
\left(\partial_{x x}+\partial_{z z}\right) \bar{\varphi}=\beta\left[\frac{\kappa-1 / 2}{\kappa-3 / 2} \bar{\varphi}-\delta_{1} \bar{n}\right]
$$

We assume that the perturbation is of the form expi $\left(k_{x} x+k_{z} z-\omega t\right)$, where $k_{x}$ and $k_{z}$ are the wave numbers in $x$ and $z$ directions, respectively. Hence, the dispersion relation for ion acoustic wave can been obtained as

$$
\begin{aligned}
& \beta_{4}\left(i \omega(v+i \omega)^{3}+k^{2}(v+i \omega)^{2} \gamma+i \omega(v+i \omega)\right. \\
& +\gamma k_{z}^{2}-\delta_{1} k^{2}(v+i \omega)^{2}-\delta_{1} k_{z}^{2}=0 \\
& \left(\gamma v^{2} k^{2}-\left(\gamma \omega^{2}-\gamma\right) k_{z}^{2}-3 v^{2} \omega^{2}+\omega^{4}-\omega^{2}\right) \\
& -\delta_{1}\left(\left(v^{2}+\omega^{2}\right) k^{2}-k_{z}^{2} / \beta_{4}+i\left(2 \gamma v \omega k^{2}\right.\right. \\
& \left.\left.+v^{3} \omega-3 v \omega^{3}+v \omega\right)-2 \delta_{1} v \omega k^{2}\right)=0
\end{aligned}
$$

where $\beta_{4}=k^{2} / \beta+\frac{\kappa-1 / 2}{\kappa-3 / 2}$. By assuming $\omega=\omega_{r}+i \omega_{i}$, the above equation reads as

$$
\begin{aligned}
X+ & i Y=0, \text { where } \\
X= & \beta_{4}\left(\left(1+3 v^{2}\right) \lambda+\gamma k_{z}^{2}+\gamma k^{2}\left(v^{2}+\lambda\right)\right. \\
& \left.-\omega_{i} v\left(v^{2}+1-6 \omega_{r}^{2}+3 \lambda+2 \gamma k^{2}\right)\right) \\
& +\delta_{1} k^{2}\left(v^{2}-2 v \omega_{i} \lambda\right)-\delta_{1} k_{z}
\end{aligned}
$$

and

$$
\begin{aligned}
Y= & -2 \omega_{r} \omega_{i} k^{2}\left(1+3 v^{2}\right)\left(\gamma+\delta_{1}\right) \\
& +\beta_{4}\left[v^{3} \omega_{r}-3 v \omega_{r}^{3}+9 v \omega_{r} \omega_{i}^{2}\right. \\
& +v \omega_{r}\left(1+2 \gamma k^{2}\right)+2 \delta_{1} v \omega_{r} k^{2}
\end{aligned}
$$

Here $\lambda=\omega_{i}^{2}-\omega_{r}^{2}$ introducing a new variable $\eta=l_{x} x+l_{z} z-M t$, where $l_{x}$ and $l_{z}$ are directional cosines and $\mathrm{M}$ is the Mach number. Equations (5)-(9) can be written as ordinary differential equations in terms of $\eta$ :

$$
\begin{aligned}
& \mathrm{M}(1-\mathrm{n})+\mathrm{n}\left(1_{\mathrm{x}} v_{x}+l_{z} v_{z}\right)=0 \\
& -M \frac{d v_{x}}{d \eta}+\left(v_{x} l_{x} \frac{d}{d \eta}+v_{z} l_{z} \frac{d}{d \eta}\right) v_{x} \\
& =-l_{x} \frac{d \varphi}{d \eta}+v_{y}-\gamma l_{x} \frac{d n}{n d \eta}+v v_{x} \\
& -M \frac{d v_{y}}{d \eta}+\left(v_{x} l_{x} \frac{d}{d \eta}+v_{z} l_{z} \frac{d}{d \eta}\right) v_{y} \\
& =-v_{x} \gamma \frac{d n}{n d \eta}+v v_{y} \\
& -M \frac{d v_{y}}{d \eta}+\left(v_{x} l_{x} \frac{d}{d \eta}+v_{z} l_{z} \frac{d}{d \eta}\right) v_{y} \\
& =-v_{x} \gamma \frac{d n}{n d \eta}+v v_{y} \\
& -M \frac{d v_{z}}{d \eta}+\left(v_{x} l_{x} \frac{d}{d \eta}+v_{z} l_{z} \frac{d}{d \eta}\right) v_{z} \\
& =-l_{z} \frac{d \varphi}{d \eta}-\gamma l_{z} \frac{d n}{n d \eta}+v v_{z}
\end{aligned}
$$

Substituting Eq. (19) in Eq. (20), we have:

$$
\frac{M}{n} \frac{d v_{x}}{d \eta}=l_{x} \frac{d \varphi}{d \eta}-v_{y}+\gamma l_{x} \frac{d n}{n d \eta}+v v_{x}
$$

Using Eq. (19), Eqs. (21) and (20) are rewritten as:

$\frac{M}{n} \frac{d v_{y}}{d \eta}=v_{x}+v v_{y}$

$\frac{M}{n} \frac{d v_{z}}{d \eta}=l_{z} \frac{d \varphi}{d \eta}+\gamma l_{z} \frac{d n}{n d \eta}+v v_{z}$

$G(\varphi)=\int_{0}^{\varphi} n d \varphi$ 
$v_{z}=b_{1} \varphi+b_{2} \varphi^{2}+b_{3} \varphi^{3}+\ldots$

Considering Eq. (23), we get:

$$
\begin{aligned}
& \frac{1}{2}\left(l_{x}^{2}+l_{z}^{2}\right)\left(\frac{d \varphi}{d \eta}\right)^{2}= \\
& \beta\left[1+\varphi+\frac{\kappa-1 / 2}{2(\kappa-3 / 2)} \varphi^{2}-\delta_{1} G(\varphi)+\delta_{2} \varphi\right]
\end{aligned}
$$

Comparing the coefficients of equal powers of $\varphi$ in Eqs. (26) and (28), up to the second order, one can obtain:

$$
\begin{aligned}
& \left(\frac{1}{3} \frac{\left(-2 \gamma a_{1} l_{z}+M b_{1}-l_{z}\right) A_{2}}{\sqrt{A_{1}}}+\left(-6 \gamma a_{2} l_{z}+2 M b_{2}\right.\right. \\
& \left.\left.-2 a_{1} l_{z}\right) \sqrt{A_{1}}-2 a_{1} v b_{1}-v b_{2}\right) \varphi^{2}+\left(\left(-2 \gamma a_{1} l_{z}\right.\right. \\
& \left.\left.+M b_{1}-l_{z}\right)+\sqrt{A_{1}}-v b_{1}\right) \varphi=0
\end{aligned}
$$

\section{III.PSEUdO-POTENTIAL APPROACH}

The Eq. (29) can be written as

$$
\frac{1}{2}\left(\frac{d \varphi}{d \eta}\right)^{2}+V(\varphi)=0
$$

The above equation is an "energy integral" of an oscillatory particle with unit mass, pseudovelocity $\frac{d \varphi}{d \eta}$ at the pseudo-position $\varphi$ in a pseudo-potential well $V(\varphi)$. To obtain an equation for $G(\varphi)$, one can expand $G(\varphi)$ and $v_{x}$ in terms of $\varphi$ :

$$
\begin{aligned}
& G(\varphi)=\varphi+a_{1} \varphi^{2}+a_{2} \varphi^{3}+\ldots \\
& v_{x}=A \varphi+B \varphi^{2}+C \varphi^{3}+\ldots
\end{aligned}
$$

To find the coefficients, one can substitute the Eqs.(31) and (32) in Eq. (29) to get,

$$
\begin{aligned}
& \left(\frac{d \varphi}{d \eta}\right)^{2}=\frac{2 \beta}{l_{x}^{2}+l_{z}^{2}}\left[\left(1+\delta_{1}-\delta_{2}\right) \varphi\right. \\
& +\left(\frac{2(\kappa-1 / 2)}{\kappa-3 / 2}+4 \delta_{1} a_{1}\right) \varphi^{2} \\
& \left.+\left(\frac{\kappa^{2}-1 / 4}{(\kappa-3 / 2)^{2}}-6 \delta_{1} a_{2}\right) \varphi^{3}+\ldots\right],
\end{aligned}
$$

And up to the second order we have

$$
\begin{aligned}
& \frac{d^{2} \varphi}{d \eta^{2}}=A_{1} \varphi+A_{2} \varphi^{2}=-\frac{d V}{d \varphi} \\
& \frac{d^{2} \varphi}{d \eta^{2}}=A_{1} \varphi+A_{2} \varphi^{2}=-\frac{d V}{d \varphi}
\end{aligned}
$$

where,

$$
A_{1}=\frac{\beta}{l_{x}^{2}+l_{z}^{2}}\left[\frac{2(\kappa-1 / 2)}{\kappa-3 / 2}+4 \delta_{1} a_{1}\right]
$$

and

$$
A_{2}=\frac{\beta}{l_{x}^{2}+l_{z}^{2}}\left[\frac{\kappa^{2}-1 / 4}{(\kappa-3 / 2)^{2}}-6 \delta_{1} a_{2}\right]
$$

Eqs. (24) and (25) can be solved as

$$
\begin{aligned}
& M \frac{d v_{x}}{d \varphi} \frac{d \varphi}{d \eta}=v_{x} G^{\prime}(\varphi)+v v_{y} G^{\prime}(\varphi) \\
& v_{y}=l_{x} \frac{d \varphi}{d \eta}+l_{x} \frac{\gamma}{G^{\prime}(\varphi)} \frac{d G^{\prime}(\varphi)}{d \eta}+v v_{x}-\frac{M}{G^{\prime}(\varphi)} \frac{d v_{x}}{d \eta}
\end{aligned}
$$

Equating the coefficients in the Eqs. (36) and (37), and expansion of $\left(\frac{d \varphi}{d \eta}\right)$ and substituting in Eq. (39) one can obtains up to second order. 


$$
\begin{aligned}
& \left(\frac{10 l_{z} a_{1} b_{1}}{l_{x}}-\frac{16 M v^{2} a_{1}^{2}}{l_{x}}-\frac{3 M v^{2} a_{2}}{l_{x}}+\frac{v^{2} b_{2} l_{z}}{l_{x}}\right. \\
& +8 M a_{1} l_{x} A_{1}+\frac{8 M^{3} A_{1} a_{1}^{2}}{l_{x}}-\frac{12 M^{3} A_{1} a_{2}}{l_{x}}- \\
& \frac{2 M^{3} A_{2} a_{1}}{l_{x}}-\frac{2 l_{z} b_{2} \sqrt{A_{1}}}{l_{x}}-10 v a_{1} l_{x} \sqrt{A_{1}}- \\
& \frac{1}{3} \frac{v l_{x} A_{2}}{\sqrt{A_{1}}}+\frac{M^{2} l_{z} A_{2} b_{1}}{l_{x}}+\frac{4 M^{2} l_{z} A_{1} b_{2}}{l_{x}}+\frac{10 v^{2} a_{1} b_{1} l_{z}}{l_{x}}- \\
& 12 M \gamma a_{2} l_{x} A_{1}-2 M \gamma a_{1} l_{x} A_{2}-\frac{1}{3} \frac{b_{1} l_{z} A_{2}}{l_{x} \sqrt{A_{1}}}+ \\
& \frac{16 M^{2} v a_{1}^{2} \sqrt{A_{1}}}{l_{x}}+\frac{12 M^{2} v a_{2} \sqrt{A_{1}}}{l_{x}}-\frac{4 l_{z} a_{1} b_{1} \sqrt{A_{1}}}{l_{x}}+ \\
& 16 \gamma v a_{1}^{2} l_{x} \sqrt{A_{1}}+6 \gamma v a_{2} l_{x} \sqrt{A_{1}}-12 M \gamma a_{1}^{2} l_{x} A_{1}+ \\
& \frac{4 M^{2} l_{z} a_{1} b_{1} A_{1}}{l_{x}}+\frac{4}{3} \frac{M^{2} v a_{1} A_{2}}{l_{x} \sqrt{A_{1}}}+\frac{2}{3} \frac{\gamma v a_{1} l_{x} A_{2}}{\sqrt{A_{1}}}+ \\
& \left.\frac{l_{z} b_{2}}{l_{x}}-\frac{16 M a_{1}^{2}}{l_{x}}+M l_{x} A_{2}-\frac{3 M a_{2}}{l_{x}}\right) \varphi^{2}+ \\
& \left(-\frac{2 M a_{1}}{l_{x}}-\frac{2 M v^{2} a_{1}}{l_{x}}+2 \gamma v a_{1} l_{x} \sqrt{A_{1}}-\frac{2 M^{3} a_{1} A_{1}}{l_{x}}+\right. \\
& \frac{4 M^{2} v a_{1} \sqrt{A_{1}}}{l_{x}}+\frac{M^{2} l_{z} b_{1} A_{1}}{l_{x}}+M l_{x} A_{1}-2 M \gamma a_{1} l_{x} A_{1}+ \\
& \left.\frac{v^{2} b_{1} l_{z}}{l_{x}}-\frac{b_{1} l_{z} \sqrt{A_{1}}}{l_{x}}+\frac{b_{1} l_{z}}{l_{x}}-v l_{x} \sqrt{A_{1}}\right) \varphi=0
\end{aligned}
$$

The coefficients $a_{1}, a_{2}, b_{1}$ and $b_{2}$ can be determined by solving the Eqs. (30) and (39).

\begin{tabular}{|c|c|c|c|c|c|}
\hline $\begin{array}{c}\text { Plasma } \\
\text { parameters }\end{array}$ & $\beta$ & $\delta_{1}$ & $\begin{array}{c}\text { Mach } \\
\text { number } \\
(\mathrm{M})\end{array}$ & $\begin{array}{c}\text { Directio } \\
\text { nal } \\
\text { cosines } \\
\left(l_{z}\right)\end{array}$ & $\begin{array}{l}\text { Spectr } \\
\text { al } \\
\text { index } \\
(\kappa)\end{array}$ \\
\hline $\begin{array}{l}\text { Range of } \\
\text { Values }\end{array}$ & $\begin{array}{l}0.46, \\
0.49, \\
0.55\end{array}$ & $\begin{array}{l}1.54, \\
2.54, \\
3.54\end{array}$ & $\begin{array}{l}2, \quad 2.1, \\
2.25\end{array}$ & $\begin{array}{l}0.1,0.4 \\
0.58\end{array}$ & $5 / 2$ \\
\hline
\end{tabular}
From equation (34), as like as [14], we get:

$$
\varphi=-\frac{3 A_{1}}{2 A_{2}} \sec h^{2}\left(\sqrt{A_{1}} \eta / 2\right)
$$

\section{IV.NUMERICAL INVESTIGATIONS}

The above results i.e. Eqs. (35)-(41), show that all coefficients are dependent to the spectral index and all plasma parameters. So for investigating the plasma parameters effects on the wave propagation, we use parameters written in Table 1:

$$
\beta=\frac{r_{g}^{2}}{\lambda_{e}^{2}}, \delta_{1}=\frac{n_{i 0}}{n_{e 0}}
$$

The variation of the pseudo-potential versus ion to electron density ratio is shown in Fig. 1. It is seen that the amplitude of $V(\varphi)$ increases with increasing the density ratio. It is concluded that increasing the ion density can lead to higher amplitude waves and also it can be said that for all positive values of $\varphi, V(\varphi)$ is positive and vice versa.

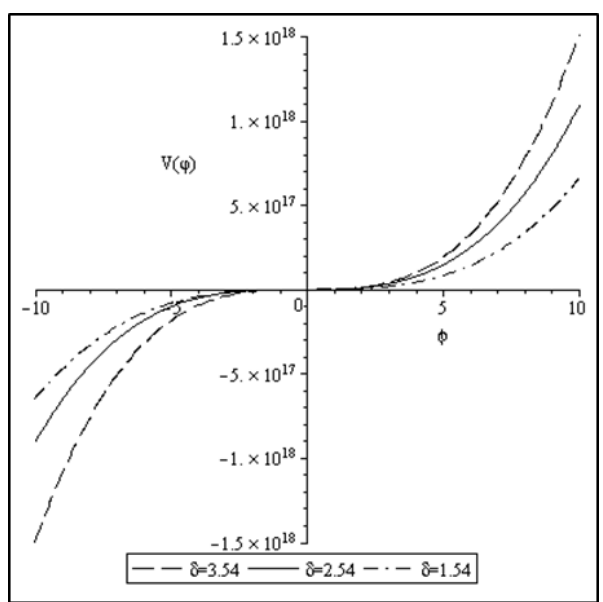

Fig. 1 Variation of Sagdeev potential with plasma potential for different values of $\delta_{1}$ and for $l_{z}=0.1$, $M=2.25$, and $\beta=0.46$.

From Fig. 2, it is concluded that the behavior of the pseudo-potential is the same for all $\beta$ values and only their amplitudes increases slightly. In other words, it can be said that the behavior of the potential is linearly dependent to the electron temperature.

In Fig. 3, the pseudo-potential is plotted as a function of $\varphi$. It is clear that for fixed value of the $\varphi$, the amplitude of the Sagdeev potential is increasing with $l_{z}$. 


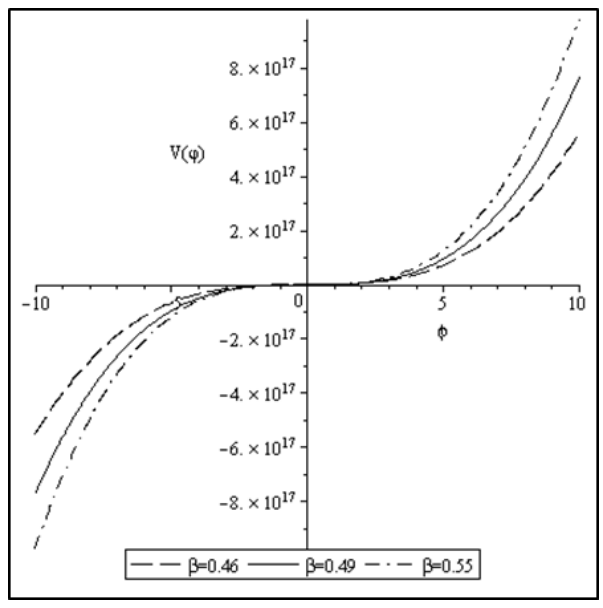

Fig. 2 The plot of $V(\varphi)$ vs. $\varphi$, for $l_{z}=0.1$, $M=2.25$, and $\delta_{1}=2.54$ for different values of $\beta$.

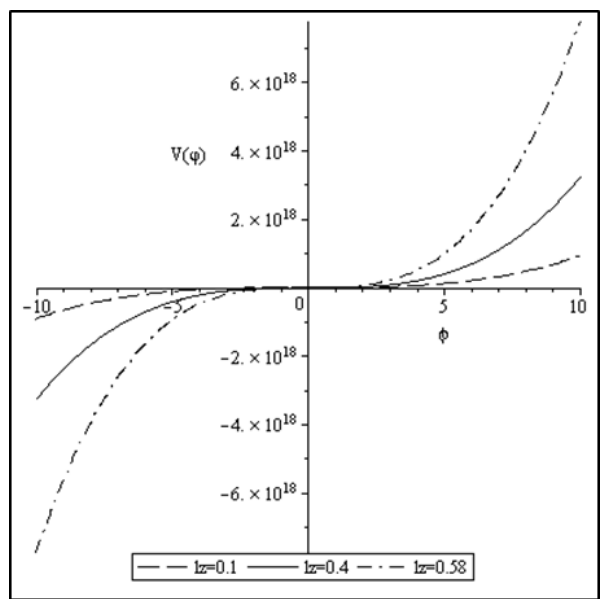

Fig. 3 The plot of $V(\varphi)$ vs. $\varphi$, for $M=2.25$, $\delta_{1}=2.54$, and $\beta=0.46$ for different values of $l_{z}$.

Figure 4 shows the plot of pseudo-potential amplitude versus plasma potential for different values of Mach number.

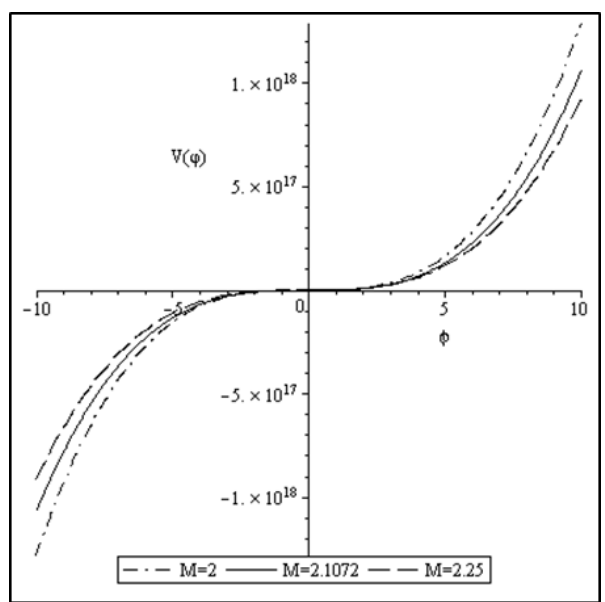

Fig. 4 The plot of $V(\varphi)$ vs. $\varphi$, for $l_{z}=0.1$, $\delta_{1}=2.54$, and $\beta=0.46$ for different values of $M$.
In Fig. 5 the pseudo-potential, $\varphi$ is plotted versus the $\eta$, for $l_{z}=0.1, M=2.25$, and $\beta=0.46$ for different values of $\delta_{1}$. It is concluded that increasing the $\delta_{1}$ may increase the plasma amplitude. Also this is negative for negative values of $\varphi$, and vice versa. Figure 6 shows that for all values of $\delta_{1}$, i.e. the ratio of ion density to the equilibrium electron density, the plasma potential is negative. It is also seen that the amplitude and the width of the potential increases as $\delta_{1}$ increases. Comparing the results for high magnitudes of $\delta_{1}$, it can be said that the slope of the amplitude is decreased with increasing the ion density.

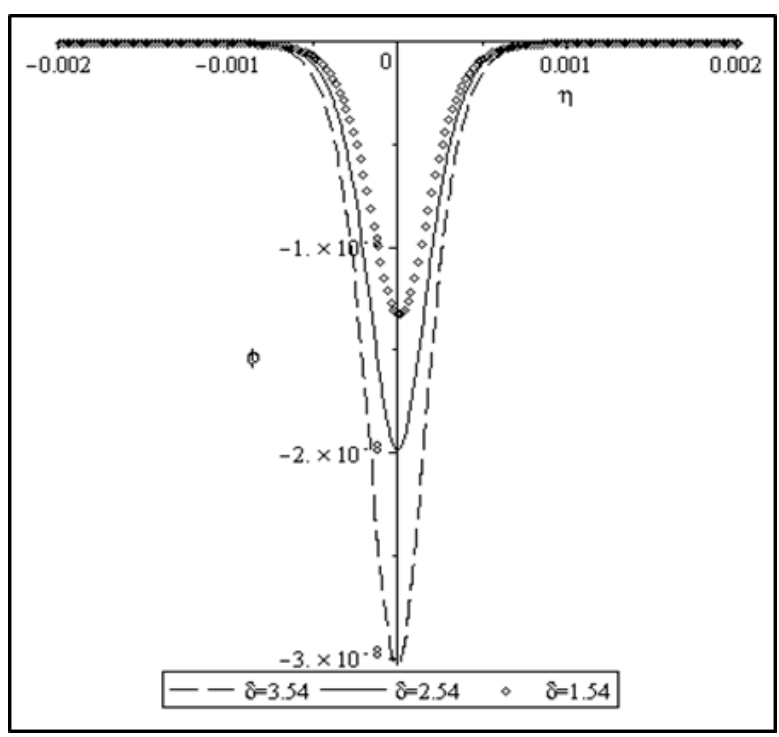

Fig. 5 The variation of $\varphi$ vs. $\eta$, for $l_{z}=0.1$, $M=2.25$, and $\beta=0.46$ for different values of $\delta_{1}$.

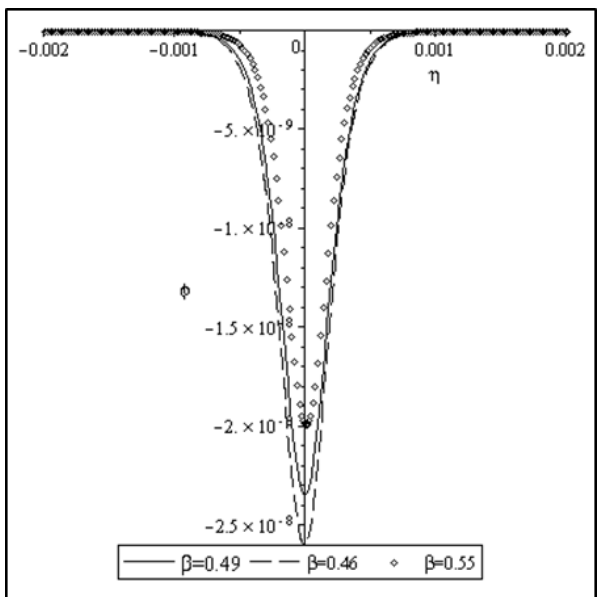

Fig. 6 The plot $\varphi$ vs. $\eta$, for $l_{z}=0.1, M=2.25$, and $\delta_{1}=2.54$ different values of $\beta$. 
The variation of plasma potential is plotted as a function of $\eta$ for $l_{z}=0.1, M=2.25$, and $\delta_{1}=2.54$ for different values of $\beta$ in Fig. 6. It is seen that decreasing the magnitude of $\beta$ may increase the width and amplitude of the potential.

In Fig. 7, we have illustrated the variation of plasma potential as a function of $\eta$ for $M=2.25, \quad \delta_{1}=2.54$, and $\beta=0.46$ for different values of $l_{z}$ and for values of $\delta_{1}$. It is seen that the amplitude is negative and that the negative amplitude and the width of the potential are increased by increasing $l_{z}$.

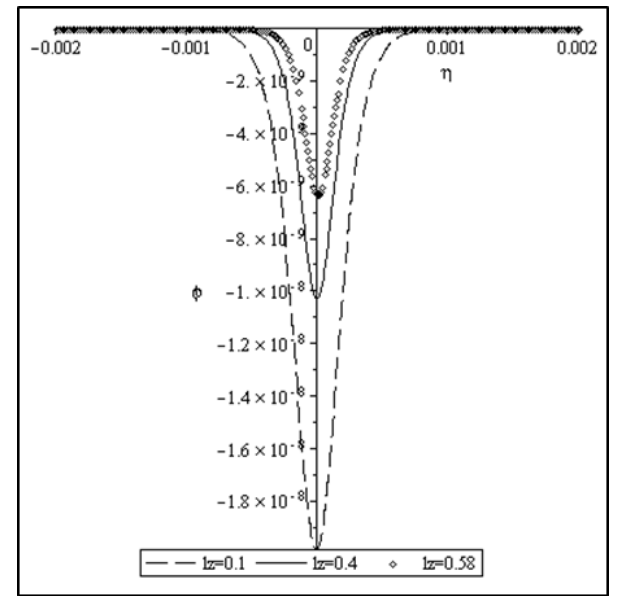

Fig. 7 The plot of $\varphi$ vs. $\eta$, for $M=2.25$, $\delta_{1}=2.54, \beta=0.46$ for different values of $\mathrm{l}_{\mathrm{z}}$.

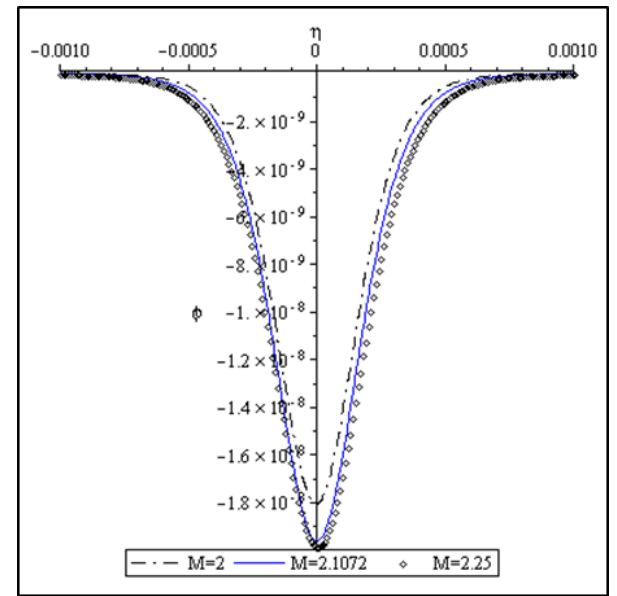

Fig. 8 The plot of $\varphi$ vs. $\eta$, for $l_{z}=0.1, \delta_{1}=2.54$, and $\beta=0.46$ for different values of $M$.

In Fig. 8, we have illustrated the variation of plasma potential as a function of $\eta$ for $l_{z}=0.1$, $\delta_{1}=2.54$, and $\beta=0.46$ for different values of Mach number.

\section{CONClusion}

Using Sagdeev potential, the dispersion relation of dust ion acoustic wave in a collisional magnetized dusty plasma is obtained in the presence of warm ions and hot electrons. It is shown that the amplitude and the width of the wave is dependent on the plasma parameters such as plasma particles density and temperatures, plasma potential, Mach number and etc. The behavior of the amplitude and the width of the wave in terms of all of these parameters is similar to that in our previous work [14]. However, the values of these two variables are different. It is shown that the Sagdeev potential has negative value for positive plasma potential and vice versa. We have also shown that increasing $\delta_{1}$ and decreasing $\beta$ can lead to the increasing of wave amplitude and also they can cause an increase in the width and the amplitude of the potential. Finally, it is found that the spectral index, i.e. the quantity when goes to infinite, the kappa distribution changes to Maxwellian one, has little effect on the propagation properties of the wave.

\section{REFERENCES}

[1] M. Alam, M. Masud, and A. Mamun, "Effects of two-temperature superthermal electrons on dust-ion-acoustic solitary waves and double layers in dusty plasmas," Astrophysics Space Sc., vol. 349, pp. 245-253, 2014.

[2] S. El-Labany, E. El-Shamy, and S. ElWarraki, "Effects of two-temperature electrons, external oblique magnetic field, and higher-order nonlinearity on dust acoustic solitary waves in a dusty plasma with vortexlike ion distribution," Astrophysics Space Sc., vol. 315, pp. 287-295, 2008.

[3] M. Hossain, A. Mamun, and K. Ashrafi, "Cylindrical and spherical dust ion-acoustic Gardner solitons in a quantum plasma," Phys. Plasmas, vol. 18, pp. 103704 (1-6), 2011.

[4] S. Popel, A. Golub, T. Losseva, A. Ivlev, S. Khrapak, and G. Morfill, "Weakly dissipative dust-ion-acoustic solitons," Phys. Rev. E, vol. 67, pp. 056402 (1-5), 2003. 
[5] N. Kundu, M. Masud, K. Ashrafi, and A. Mamun, "Dust-ion-acoustic solitary waves and thezir multi-dimensional instability in a magnetied nonthermal dusty electronegative plasma," Astrophys. Space Sci. vol. 343, pp. 279-287, 2013.

[6] A. Mamun, N. Jahan, and P. Shukla, "DIA and DA solitary waves in adiabatic dusty plasmas," J. Plasma Phys. vol. 75, pp. 413431, 2009.

[7] T.K. Baluku and M.A. Hellberg, "Ion acoustic solitons in a plasma with two-temperature kappa-distributed electrons," Phys. Plasmas, vol. 19, pp. 012106 (1-10), 2012.

[8] A. Saha and P. Chatterjee, "New analytical solutions for dust acoustic solitary and periodic waves in an unmagnetized dusty plasma with kappa distributed electrons and ions," Phys. Plasmas, vol. 21, pp. 02211 (1-6), 2014.

[9] T.K. Baluku and M.A. Hellberg, "Dust acoustic solitons in plasmas with kappadistributed electrons and/or ions," Phys. Plasmas, vol. 15, pp. 123705 (1-11), 2008.

[10] M.A. Hellberg, R.L. Mace, T.K. Baluku, I. Kourakis, and N. Saini, "Comment on "Mathematical and physical aspects of Kappa velocity distribution"'[Phys. Plasmas14, 110702 (2007)]," Phys. Plasmas, vol. 16, pp. 094701 (1-9), 2009.

[11]G. Banerjee and S. Maitra, "Pseudopotential approach for dust acoustic solitary waves in dusty plasmas with kappa-distributed ions and electrons and dust grains having power law size distribution," Phys. Plasmas (1994present), vol. 22, pp. 043708-9, 2015.

[12] N. Saini, N. Kaur, and T.S. Gill, "Dust acoustic solitary waves of KadomstevPetviashvili (KP) equation in superthermal dusty plasma," Advances in Space Research, vol. 55, pp. 2873-2882, 2015.

[13] M. Michael, S. Gopinathan, S. Sebastian, N. T. Willington, A. Varghese, R. Gangadharan, and Ch. Venugopal "Kadomstev-PetviashvilliBurgers (KPB) Equation in a Five Component Cometary Plasma with Kappa Described Electrons and Ions," J. Adv. Medic. Education Profession., vol. 3, pp. 1431-1442, 2015.

[14] M. R. Sayyar, H. Zahed, S. J. Pestehe, and S. Sobhanian, "Propagation of arbitrary amplitude dust-ion acoustic waves in a collisional magnetized dusty plasma in the presence of non-thermal electrons", Phys. Plasmas, vol. 23, pp. 73704 (1-6), 2016.

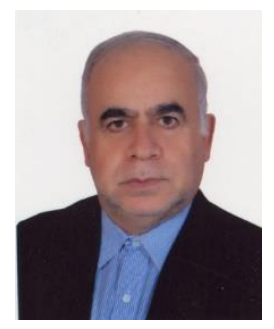

Samad Sobhanian, Professor Emeritus in plasma physics, from University of Tabriz, is graduated from University of Paul Sabatier, Toulouse, France in atomic collisions in plasma. His main research works include both theoretical and experimental plasma physics. $\mathrm{He}$ is a member of Iran Nanotechnology, Physics society of Iran and Optics and Photonics Society of Iran.

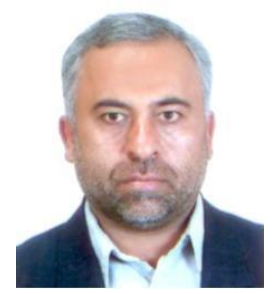

Hossein Zahed, associate professor in plasma physics, from Sahand University of Technology, is graduated from University of Tabriz in plasma. His main research works is theoretical plasma physics. He is a member of Iran Nanotechnology, Physics Society of Iran and Optics and Photonics Society of Iran.

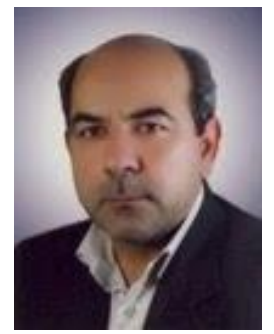

Sayyed Jalal Pestehe is associated professor of atomic and molecular department of the University of Tabriz. He completed his B.Sc. and M.Sc. and Ph.D. studies at the Universities of Tabriz and York, respectively. He has supervised 24 M.Sc. and 3 Ph.D. thesis since 2002 and published nearly 80 papers in journals and conference proceedings. His 
recent work focuses on the low temperature plasmas, nanothechnology, and dusty plasmas. $\mathrm{He}$ is a member of Iran nanotechnology, Physics Society of Iran and Optics and Photonics Society of Iran.

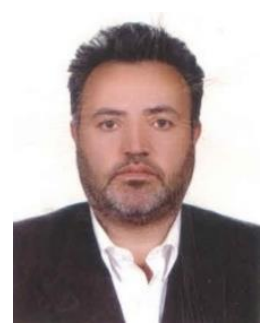

Mohammad Reza Sayyar, assistant professor of Plasma Physics in physics department of the
Maragheh University, $\mathrm{He}$ is graduated from University of Tabriz in plasma physics. He is a member of Iran Nanotechnology, Physics Society of Iran and Optics and Photonics Society of Iran. 
THIS PAGE IS INTENTIONALLY LEFT BLANK. 